\title{
INTERCAMBIO DE ALUMNOS. UNA EXPERIENCIA DE INTERNACIONALIZACIÓN DE LA EDUCACIÓN
}

\author{
Nuria Alsina"
}




\section{RESUMEN}

Este artículo recoge la experiencia desarrollada por la Pontificia Universidad Católica de Chile (PUC) en el intercambio estudiantil internacional. Analiza el efecto que este proceso ha tenido, tanto en la formación de los alumnos que han participado, como en el enriquecimiento de la docencia propia gracias a la presencia de alumnos extranjeros.

Analiza, muy brevemente, el efecto multiplicador que, como consecuencia de los convenios de intercambio, ha favorecido a otros quehaceres de la universidad y, finalmente, señala su evolución a través de los datos estadísticos más relevantes.

\section{ABSTRACT}

This paper takes up the experience developed by the Pontificia Universidad Católica de Chile (PUC) in international students' exchange. It analyses the effect that this process has had regarding not only the pupils taking part in it, but also the teaching enrichment, due to the presence of foreign visitors.

It explains, very briefly, the multiplying effect that has favoured other tasks, as a consequence of the interchange's agreements, and, finally, it points out its evolution through the most relevant statistic data. 


\section{INTERCAMBIO DE ALUMNOS. \\ UNA EXPERIENCIA DE INTERNACIONALIZACIÓN \\ DE LA EDUCACIÓN}

\section{INTRODUCCIÓN}

De un estudio realizado en ciento ochenta y tres universidades norteamericanas se concluyó que los principales factores de internacionalización en las universidades son: competencia internacional de los profesores, apoyo de la gestión central y una adecuada asignación de recursos.

La combinación de estos factores y una oportuna gestión central han permitido a la Pontificia Universidad Católica de Chile (PUC) desarrollar un amplio programa de intercambio de alumnos que ha tenido muchos beneficios, no sólo para los alumnos directamente involucrados sino también para otras actividades académicas, tales como: el intercambio de profesores, la participación en proyectos que operan a través de la participación en redes y, recientemente, estudios de doble titulación en los ámbitos de pre y posgrado.

El programa de intercambio de alumnos puede describirse a través de dos vertientes:

\section{ALUMNOS EXTRANJEROS DE INTERCAMBIO}

Nace formalmente en 1990 gracias a la posibilidad de ofrecer a un grupo de alumnos de la Universidad de Stanford un programa especial de un trimestre de duración enfocado en estudios de América Latina que incluía cursos en Economía, Historia, Ciencias Políticas y Sociología, además de cursos de español en tres niveles. La experiencia fue muy enriquecedora y se decidió explorar esta oportunidad, nueva en Chile, y extenderla a otras universidades extranjeras ofreciendo programas en distintas modalidades y con un criterio de flexibilidad y calidad. 
La universidad no estaba preparada para acometer este programa, dada la flexibilidad requerida en aspectos como: tiempos de duración, plazos de postulación, prerrequisitos, fechas de inscripción y registros en cursos, nivel de idioma, y otros: formalidades propias de una universidad muy ordenada y estructurada, pero orientada a las necesidades de alumnos regulares. La experiencia que existía era recibir alumnos extranjeros de posgrado, pero que, al efectuar sus programas completos en la PUC, tenían la categoría de alumno regular y se regían por esa reglamentación.

Fue necesario trabajar estrechamente con las distintas unidades administrativas para crear condiciones especiales que permitieran recibir, formalmente, a los extranjeros y ofrecer los mismos servicios a los que tenían acceso los alumnos regulares. Un adecuado trabajo conjunto con las distintas unidades académicas fue también fundamental, especialmente para la orientación y registro en cursos regulares.

Gracias a esto, el programa ha podido crecer tal como lo reflejan las cifras que se muestran más adelante. Posteriormente se abre la reciprocidad, oportunidad para que los alumnos regulares de la universidad puedan realizar una estadía de intercambio de estudios en alguna de las universidades extranjeras.

Actualmente el programa ofrece distintas modalidades.

Programas regulares: los alumnos se insertan en cursos regulares de nuestras carreras, pudiendo elegir entre una amplia gama de posibilidades y registrándose, simultáneamente, en distintas escuelas o institutos.

Como parte de estas opciones, se ofrece la posibilidad de que los alumnos cursen un grupo de asignaturas que conforman los certificados académicos o diplomas de pregrado (de 50 créditos), constituidos por cursos regulares de distintos carreras. El más atractivo ha sido el diploma o certificado académico en Estudios Latinoamericanos. Con esto se otorga un valor agregado a la estadía de intercambio.

Programas especiales ("tailor made program"): hechos a medida, en atención a los requerimientos de la universidad extranjera, en contenido tiempo de duración y período del año en que se ofrecen. 
En este tipo de programa, el criterio de flexibilidad, sin transar en la calidad de los cursos o seminarios ofrecidos, es fundamental. Un ejemplo de ello es el seminario de "Historia y Cultura Chilena, un enfoque interdisciplinario".

Programas mixtos: una combinación de los dos tipos anteriores, en que los alumnos cursan un semestre regular y reciben, simultáneamente, cursos especiales.

Programa de español para extranjeros: atendiendo a que uno de los objetivos fundamentales de los programas de intercambio es la experiencia cultural, académica y el estudio de un segundo idioma, el programa de español para extranjeros cobra mayor importancia. Este programa ofrece diversas modalidades de cursos intensivos, semestrales, tutoriales y en distintos niveles, para satisfacer las distintas necesidades de los alumnos extranjeros.

Alumnos visitantes de investigación: esta forma permite que alumnos terminales puedan acceder a profesores y a servicios regulares de la universidad en la etapa de realización de su trabajo final de tesis de pre y posgrado, sin el necesario registro en cursos.

$\mathrm{N}^{\circ}$ de alumnos extranjeros en intercambio en PUC

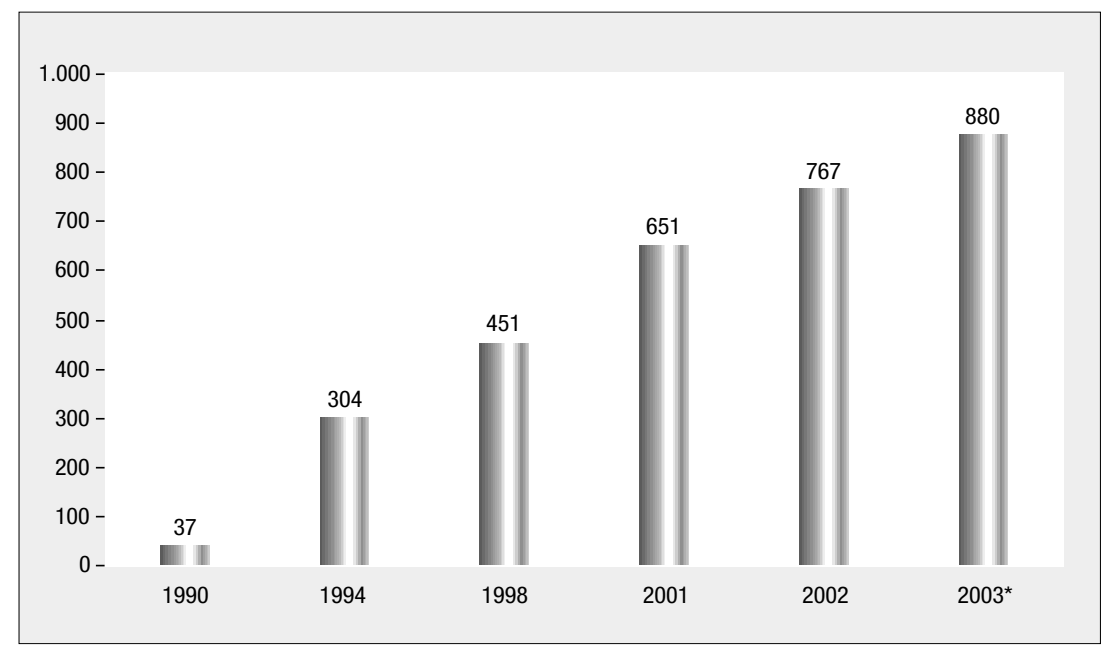

Un $10 \%$ de estas cifras corresponde a alumnos provenientes de programas de posgrado en su universidad de origen. 
$\mathrm{N}^{\circ}$ de alumnos extranjeros en intercambio en PUC según país de origen

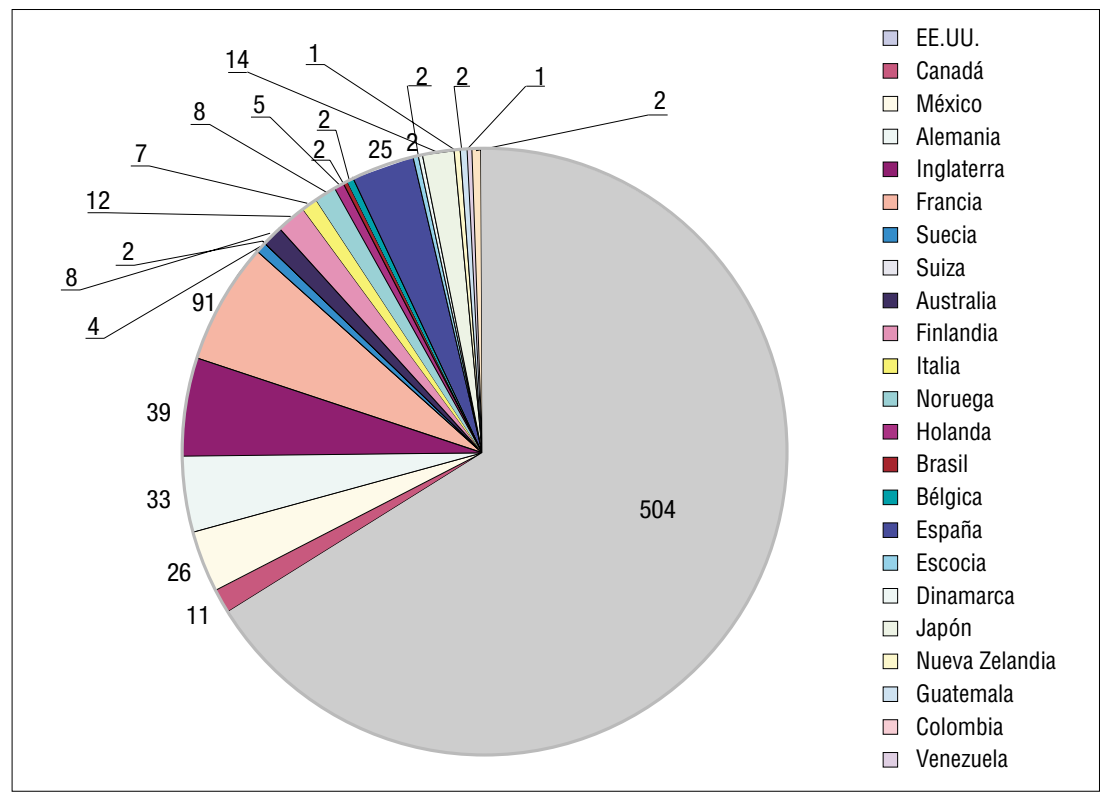

Áreas más demandadas por alumnos extranjeros en PUC

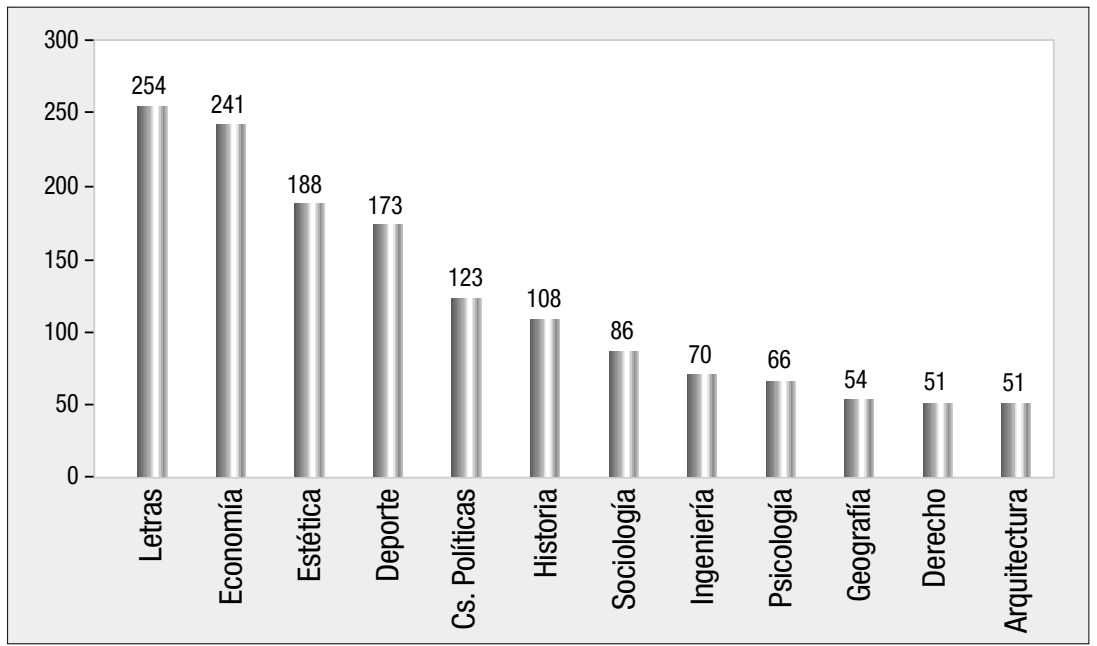

Una amplia gama de cursos, dictados a través de las treinta y dos unidades académicas organizadas en dieciocho facultades, está abierta a los alumnos extranjeros, que deben cumplir el requisito equivalente y tener un promedio de notas en el $25 \%$ superior de su escala. Sin embargo, existen áreas de mayor interés como, por ejemplo, Economía, Historia, Política, Literatura, Sociología, Arte, Medio Ambiente y Aspectos de la Cultura Chilena. 
Los alumnos extranjeros que realizan una estadía en la universidad tienen acceso a los mismos servicios que la universidad ofrece normalmente a los estudiantes regulares, sin discriminación: acceso al sistema de biblioteca, servicios de salud, laboratorios de computación, credencial universitaria, deportes y otros.

Las actividades extracurriculares, ofrecidas normalmente por la universidad, son fundamentales como instancia de integración de los alumnos chilenos y extranjeros, en un ambiente distinto al de la sala de clases.

Por otra parte, las actividades de acción social despiertan un especial interés en los extranjeros, puesto que tienen la posibilidad de acceder a actividades que regularmente organiza la PUC: los trabajos de verano y de invierno en comunidades alejadas del centro del país, por ejemplo.

\section{ALUMNOS PUC DE INTERCAMBIO EN EL EXTRANJERO}

Esta etapa del programa se inició formalmente en 1994 con el envío de un grupo de alumnos a diferentes campus de la Universidad de California. La experiencia obtenida, el grado de satisfacción de los participantes y los buenos resultados alcanzados, impulsaron a la universidad a extenderlo. Se inició, entonces, una intensa búsqueda de oportunidades de intercambio con universidades de EE.UU. y Europa, en una primera etapa; la cifra creció el segundo año y se amplió el número de universidades y países de destino. A la fecha, más de mil doscientos alumnos de pre y posgrado han realizado una estadía de intercambio en el extranjero.

El programa de estadía abarca normalmente un semestre y, excepcionalmente y como máximo, un año Los participantes mantienen su categoría de alumno regular en la PUC y cursan un semestre académico en la universidad de destino, previo un riguroso proceso de selección y postulación realizado en estrecha coordinación y según los requisitos de la institución que los acoge. Los cursos realizados en la universidad extranjera son reconocidos como parte 
del plan regular de estudios en la PUC. Por ello, en el proceso de postulación, la etapa de diseño y aprobación del plan de estudios que cursará en la universidad que lo acoja -por parte de la unidad académica de origen del alumno-, resulta fundamental para este objetivo. Recientemente, el intercambio ha posibilitado extender y enriquecer la experiencia aspirando al desarrollo de dobles titulaciones en el ámbito de pre y posgrado.

En este momento, existe doble titulación -en el MBA con la Universidad de Texas Austin y con HEC de París-, obtenida por el alumno una vez que realiza una pasantía de un año en cada universidad, convalidándole la institución extranjera lo que ha aprobado en la universidad de origen. Este programa opera en ambas direcciones. Una tercera experiencia existe en el Magíster en Educación y Comunicación con la Universidad Autónoma de Barcelona.

En la actualidad, se está estudiando dos nuevas dobles titulaciones, esta vez en el ámbito de pregrado, en Ingeniería, Economía y Ciencia Política con universidades francesas, principalmente.

$\mathrm{N}^{\circ}$ de alumnos PUC en intercambio

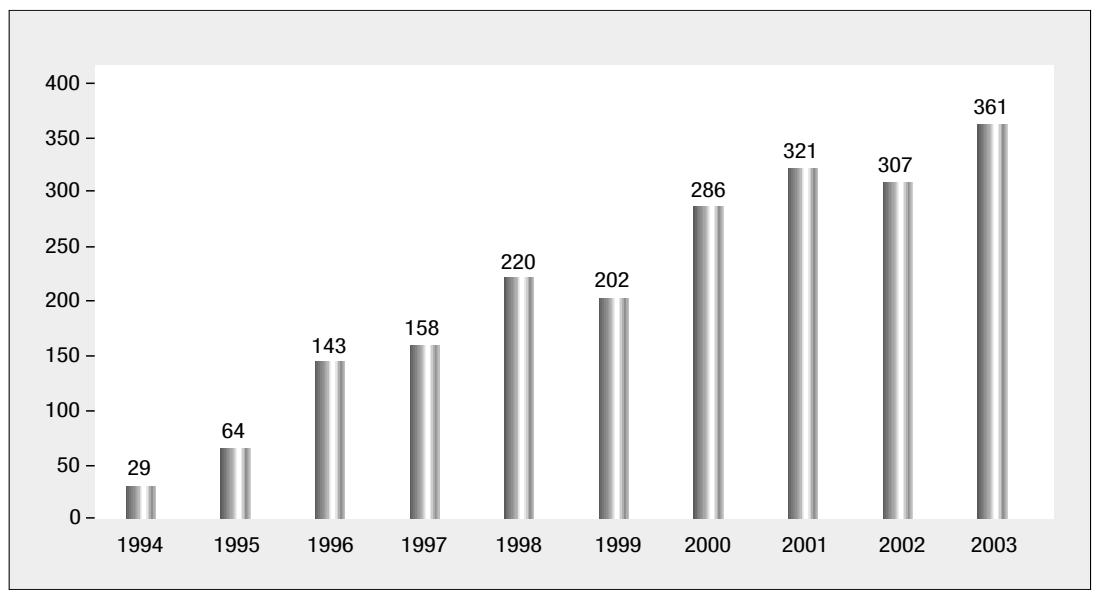


$\mathrm{N}^{\circ}$ de alumnos PUC en intercambio según país de destino

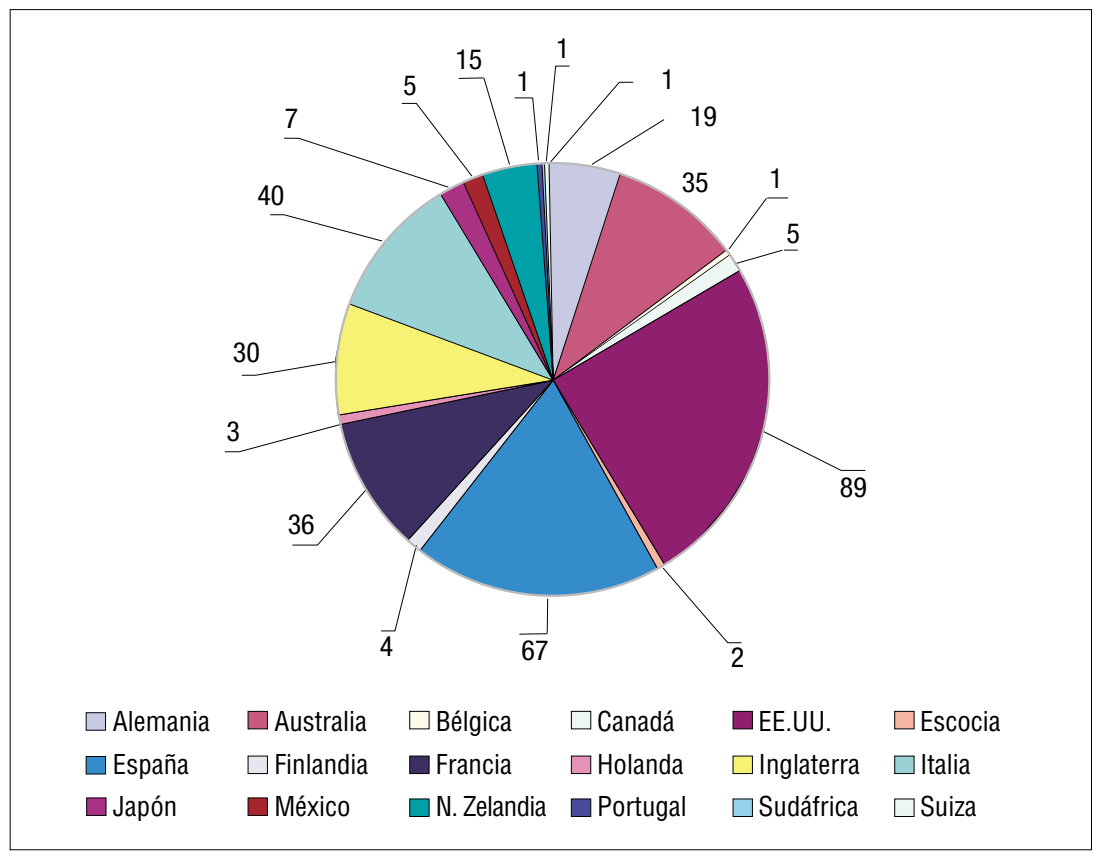

Evolución del Programa de Intercambio PUC

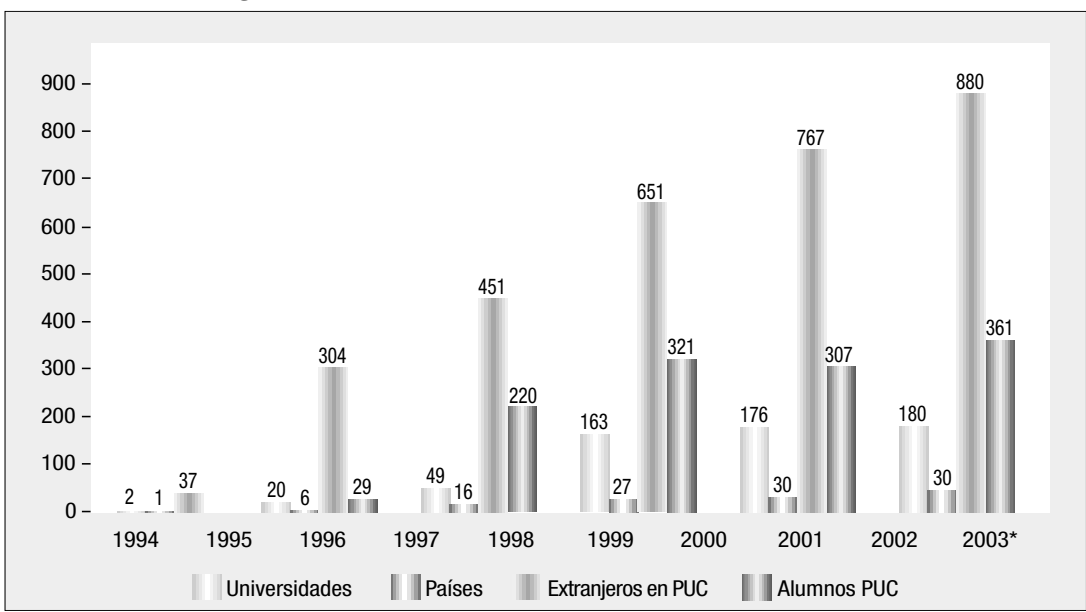

(*) Entre 1990 y 1994, 15 alumnos de Economía y Administración realizaron intercambio en universidades de Estados Unidos, como experiencia piloto. 


\section{Acuerdos de Intercambio}

Alemania

- Albert Ludwigs-Universität Freiburg

- Bayerische Julius Maximilans UniversitätWürzburg

- Eberhard Karls Universität -Tübingen

- Hochschule für Musik de Detmold

- Ludwig Maximilians Universität-München

- Ruprecht Karls Universität-Heidelberg

- Technische Universität Berlin

- Technische Universität München

- Universität Mannheim

- University of Applied Sciences Karlsruhe

\section{Argentina}

- Universidad Católica de Buenos Aires

- Universidad Nacional de San Juan

- Universidad Católica de Córdoba

Australia

- Griffith University

- Monash University

- University of New South Wales

- University of South Australia

- University of Technology-Sydney

Austria

- Akademie Der Bildenden Künste in Wien

Bélgica

- Université Catholique du Lovaine

Canadá

- Queen's University at Kingston

- Simon Fraser University

- Université de Montréal

- Université Laval

- University of Alberta

- University of British Columbia

Colombia

- Universidad de los Andes

\section{Dinamarca}

- Engineering College of Copenhagen

\section{Escocia}

- Napier University

- Robert Gordon University

- The University of Glasgow

\section{España}

- EINA

- Escola Superior de Disseney Elisava

- Institut del Teatre de la Diputació de Barcelona

- Programa INTERCAMPUS

- Universidad Autónoma de Madrid

- Universidad Carlos III de Madrid

- Universidad de Alcalá de Henares

- Universidad de Cantabria

- Universidad de Granada

- Universidad de Salamanca

- Universidad de Sevilla

- Universidad del País Vasco

- Universidad Politécnica de Cataluña

- Universidad Politécnica de Madrid

- Universidad Politécnica de Valencia

- Universidad Pontificia de Comillas

- Universidad Pontificia de Salamanca

- Universidad Pública de Navarra

- Universidad Ramón Llull

- Universidad Santiago de Compostela

- Universitat Autónoma de Barcelona

- Universitat Pompeu

Estados Unidos

- Boston College

- Carnegie Mellon University

- Duke University - School of Law

- New York University

- St. John's University

- The University of Texas at Austin

- University of California (Berkeley, UCLA, Sta. Barbara, Sta. Cruz, Irvine, Davis, Riverside, San Diego)

- University of Georgia

- University of Illinois at Urbana Champaign

- University of Maryland

- University of Miami, Coral Gables

- University of Montana

- University of North Carolina at Chapel Hill

- University of North Carolina at Charlotte

- University of Wisconsin - Madison

\section{Finlandia}

- Helsinki School of Economics and Business Administration (sólo ramos de Economía y Administración) 


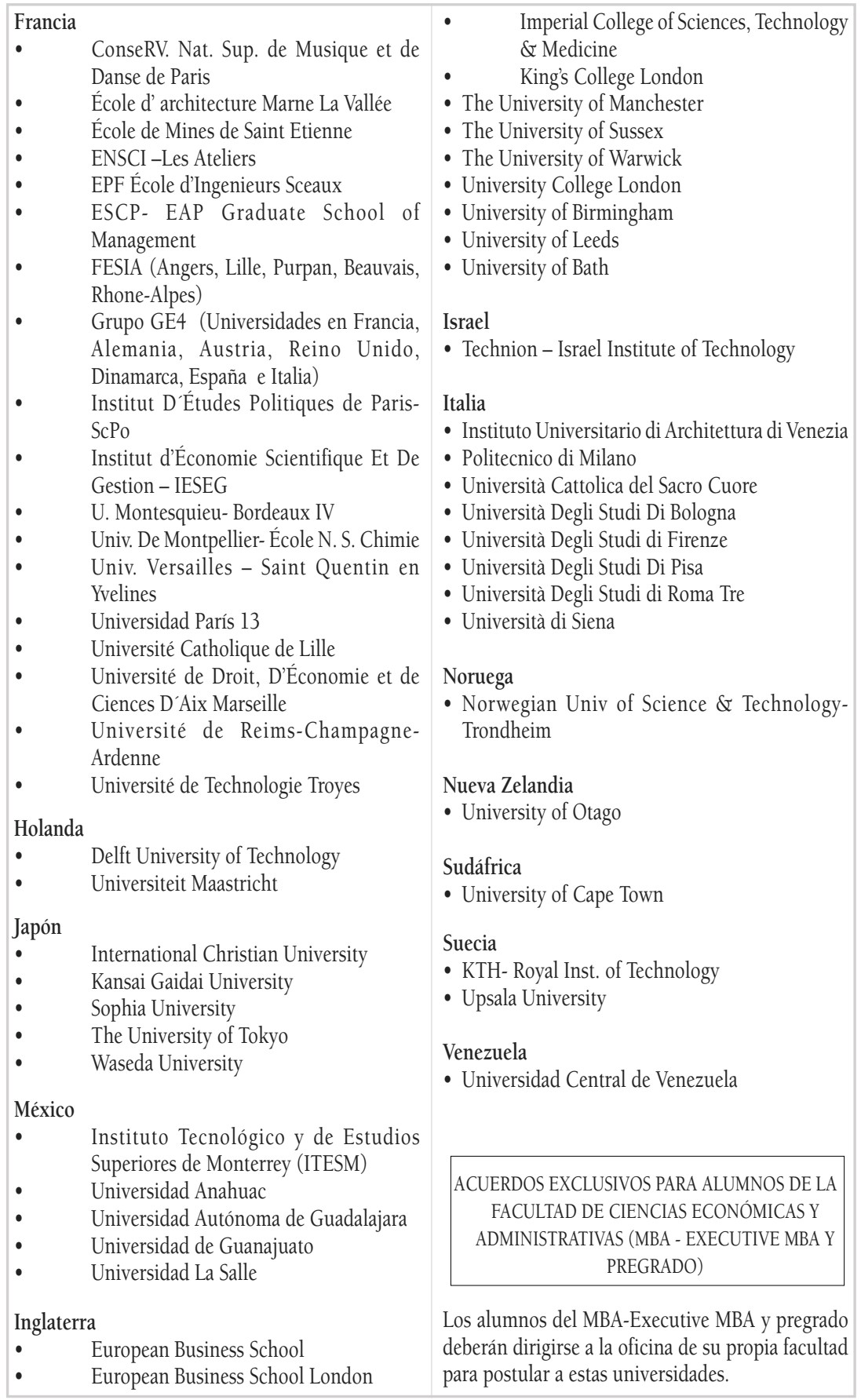




\begin{tabular}{|c|c|}
\hline 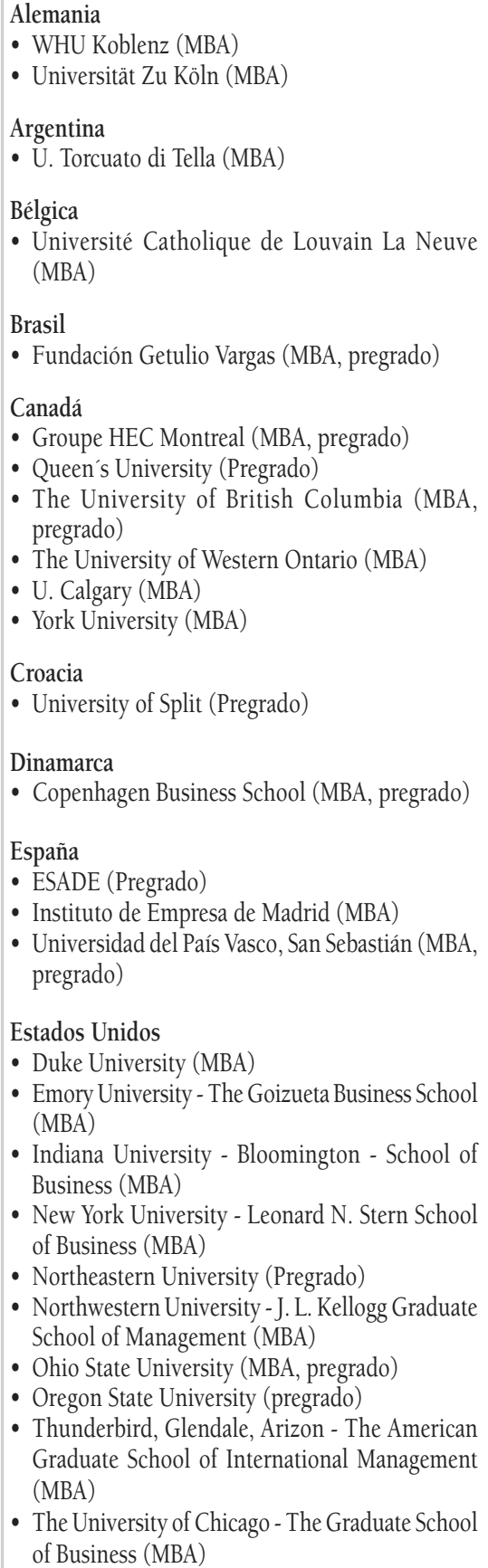 & $\begin{array}{l}\text { - University of Florida (MBA, pregrado) } \\
\text { - The University of North Carolina at Chapel Hill } \\
\text { - The Kenean-Flager Business (MBA, pregrado) } \\
\text { - The University of Texas at Austin - Graduate } \\
\text { School of Business (MBA, pregrado) } \\
\text { - The University of California, Davis-Graduate } \\
\text { School of Management (MBA) } \\
\text { - University of California UCLA - The Anderson } \\
\text { School of Management (MBA) } \\
\text { - University of Washington - Seattle - Graduate } \\
\text { School of Business (MBA) } \\
\text { - University of Wisconsin - Madison - Graduate } \\
\text { School of Business (MBA, pregrado) } \\
\text { - Vanderbilt University - Owen Graduate School } \\
\text { of Management (MBA) } \\
\text { Finlandia } \\
\text { - Jyväskyla Polytechnic University (MBA, } \\
\text { pregrado) } \\
\text { - Helsinki School of Economics and Business } \\
\text { Administration (MBA, pregrado) } \\
\text { Francia } \\
\text { - ESCP- EAP Graduate School of Management } \\
\text { (pregrado) } \\
\text { - Groupe HEC París (MBA) } \\
\text { - Groupe ESC Reims (MBA, pregrado) } \\
\text { - E.M. Lyon - École de Management (MBA, } \\
\text { - Telaviv University - The Leon Recanati Graduate } \\
\text { School of Business Administration (MBA) } \\
\text { - Université de Pau (MBA, pregrado) } \\
\text { - Université Paris Douphine (pregrado) } \\
\text { - Grenoble Graduate School of Business (pregrado) } \\
\text { Guatemala } \\
\text { - Universidad San Francisco Marroquin (MBA, } \\
\text { pregrado) } \\
\text { Inglaterra } \\
\text { Holanda } \\
\text { - De Universiteit van Amsterdam - Faculty of } \\
\text { Economics and Econometrics (MBA) } \\
\text { School of Management (MBA) } \\
\text { - Enity - Rotterdam } \\
\text { - }\end{array}$ \\
\hline
\end{tabular}




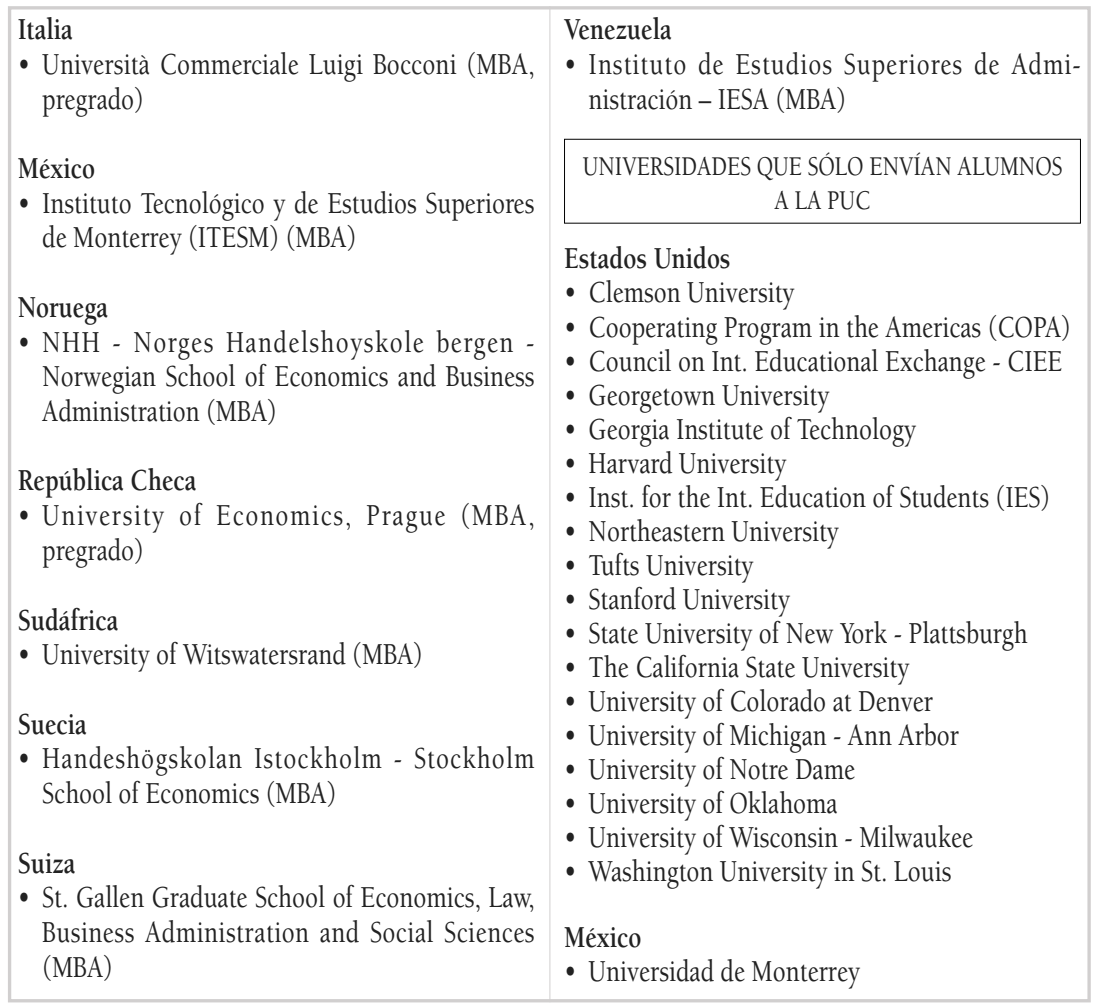

\section{ALGUNAS CONSIDERACIONES FINALES}

La experiencia de desarrollar este programa de intercambio ha sido enormemente positiva. Ha permitido que más de tres mil alumnos extranjeros - de treinta países y ciento setenta universidades- hayan realizado una estadía en nuestra universidad. Para Chile, por cierto, ha sido un importante aporte al proceso de internacionalización de la educación. Los estudiantes extranjeros enriquecen la sala de clase con su interés y actitud participativa, demandando muchas veces mayor atención de parte del profesor.

El programa:

- Ha permitido estrechar lazos con muy buenas universidades, lo que se ha redituado en intercambio de profesores y visitas de misiones para ampliar estas relaciones y participar en redes y proyectos internacionales de cooperación. 
- Ha despertado interés por parte de los alumnos chilenos en continuar estudios de posgrado en el extranjero, en otra universidad o en la misma en la que se realizó el intercambio de pregrado. Ha dado la oportunidad de conseguir, en algunos casos, condiciones especiales de matrícula para que nuestros profesores jóvenes -o alumnos egresados- continúen estudios de posgrado.

- Posibilita obtener dobles titulaciones como resultado de un intercambio más extenso y previo acuerdo específico.

- Ha prestigiado a la universidad chilena, por el buen resultado obtenido por los alumnos chilenos en las universidades de intercambio. Asimismo, los alumnos extranjeros han podido comprobar la seriedad y calidad de la enseñanza en Chile.

\section{TENDENCIAS FUTURAS}

Se espera la incorporación de más universidades chilenas a este tipo de programa.

Siguen existiendo sesgos; por una parte, por el manejo del idioma requerido para realizar un intercambio en una universidad de habla no hispana y, por otra -y sobre todo-, por el factor económico: dada la realidad chilena, muy pocos alumnos pueden cubrir los gastos de pasaje y estadía que implica el intercambio. Sólo la matrícula está cubierta por la reciprocidad.

En este sentido, la PUC ha realizado esfuerzos por conseguir becas, tanto propias como extranjeras, para que alumnos excelentes, pero de muy bajos recursos, puedan acceder a este programa. En cuanto al problema del idioma, desde hace más de seis años se organizan en la universidad cursos instrumentales para preparar a los alumnos en inglés, francés y alemán. 
Evolución de becas para intercambio 1994-2003

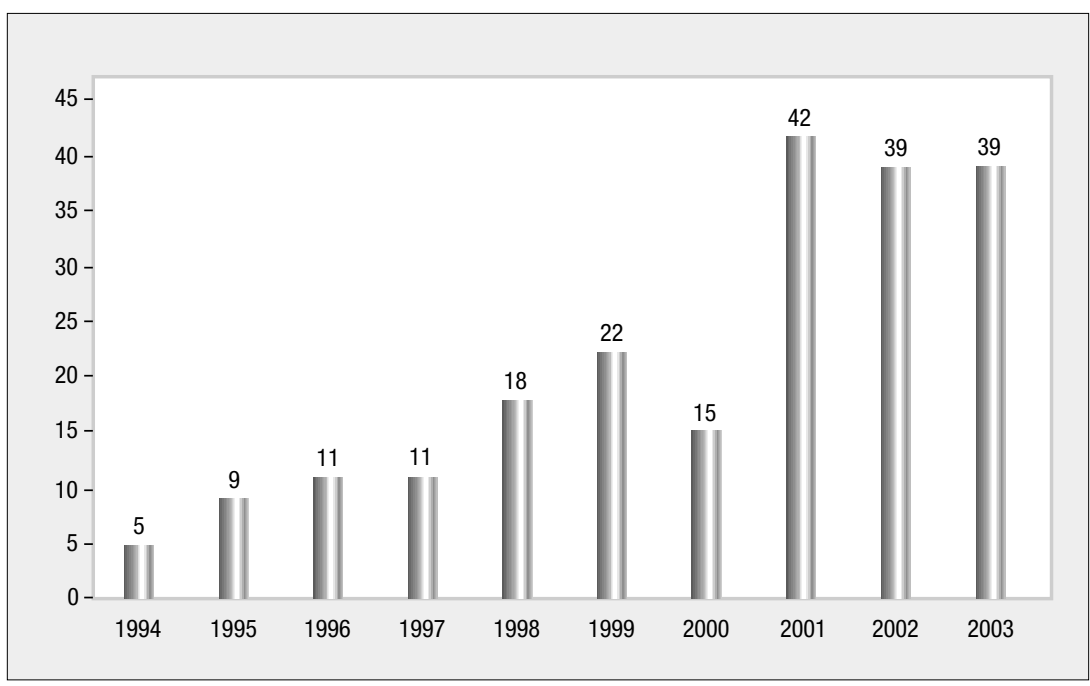

Evolución de cursos de idioma PUC

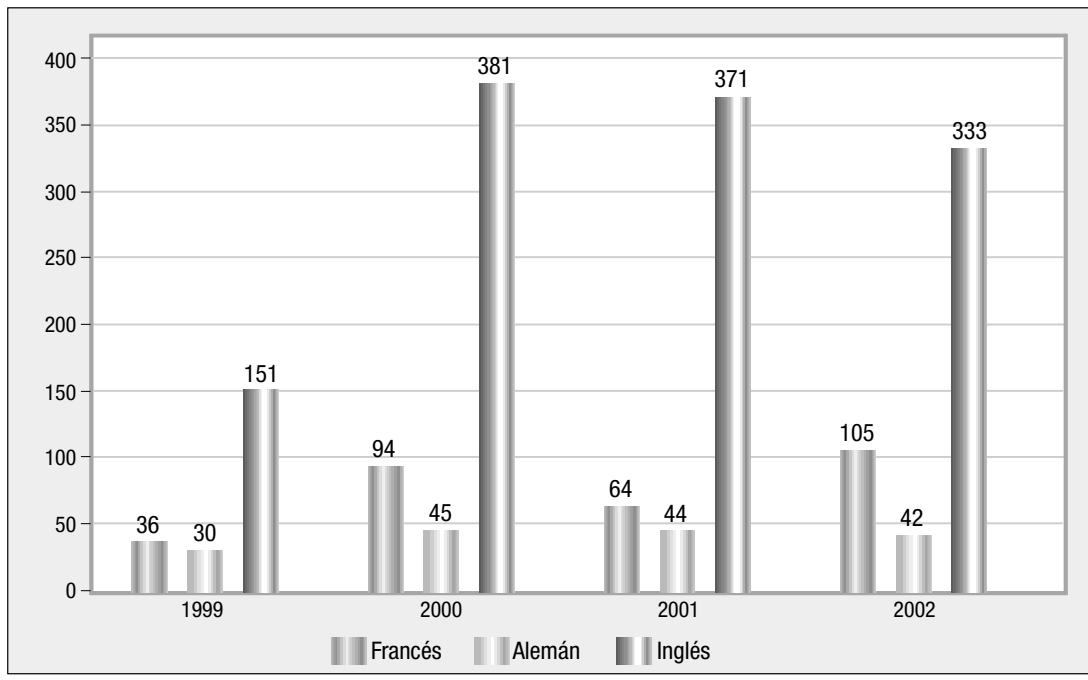

